\title{
Differential use of pulse wave velocity and ankle- brachial index in elderly Chinese and novel serum bio-markers for predicting arterial stiffness: a cross- sectional study
}

\section{Guoxiang Fu}

Shanghai Jiao Tong University affiliated Shanghai Sixth people's Hospital

\section{Yuan Zhong}

Shanghai Jiaotong University affiliated Shanghai Sixth people's Hospital

\section{Fengfeng Pan}

Shanghai Jiaotong University affiliated Shanghai Sixth people's Hospital

Chanchan Xu ( $\sim$ chanchansh@163.com)

Shanghai Jiaotong University affiliated Shanghai Sixth people's Hospital https://orcid.org/0000-00015358-3279

\section{Research article}

Keywords: Arterial stiffness in elderly, Pulse wave velocity, Ankle-brachial Index, Vascular cell adhesion molecule, Osteopontin

Posted Date: October 24th, 2019

DOI: https://doi.org/10.21203/rs.2.16405/v1

License: (c) (1) This work is licensed under a Creative Commons Attribution 4.0 International License.

Read Full License 


\section{Abstract}

Background Arterial stiffness is characterized by the loss of vessel elasticity and arterial compliance. Brachial-ankle pulse wave velocity (PWV) and ankle-brachial Index (ABI) are widely used in clinic for evaluating arterial stiffness. This study aimed to compare the efficiency of PWV and $A B I$ in elderly Chinese and investigate novel bio-markers for aging related arterial stiffness.

Methods PWV and ABI were evaluated in 169 elderly Chinese with mean age of 85.7 years. 86 of the participants had serum concentrations of angiotensin $\otimes$, vascular cell adhesion molecule (VCAM), osteopontin and glutathione peroxidase tested in further.

Results Compared with normotensive participants, PWV levels elevated in both limbs of hypertensive patients ( $2219 \pm 90 \mathrm{~cm} / \mathrm{s}$ v.s. $1970 \pm 66 \mathrm{~cm} / \mathrm{s}, \mathrm{P}=0.090$ and $2141 \pm 52 \mathrm{~cm} / \mathrm{s}$ v.s. $1932 \pm 67$ $\mathrm{cm} / \mathrm{s}, \mathrm{P}=0.023$, separately) . Compared with non- diabetes mellitus participants, $A B \mid$ levels declined in both limbs of diabetes mellitus (DM) patients $0.96 \pm 0.02$ v.s. $1.05 \pm 0.02, P=0.002$ and $0.99 \pm 0.03$ v.s. $1.03 \pm 0.02, P=0.071$, separately). VCAM was included in both regression models for predicting PWV in left limbs and PWV in right limbs (Beta $=-0.307, P=0.048$; Beta $=-0.358, P=0.020$, separately).

Osteopontin was included in both regression models for predicting $A B I$ in left limbs and $A B I$ in right limbs ( Beta $=-0.320, P=0.039 ;$ Beta $=-0.290, P=0.011$, separately).

Conclusions: Differential use of PWV and ABI should be considered in elderly with hypertension and/or DM. Serum levels of VCAM and osteopontin might be independent bio-markers for predicting arterial stiffness.

KEYWORDS Arterial stiffness in elderly; Pulse wave velocity; Ankle-brachial Index; Vascular cell adhesion molecule; Osteopontin

\section{Background}

Cardiovascular disease and cerebral vascular disease are the leading causes of mortality and morbidity worldwide.1-3

Arterial stiffness has been recognized to be involved in the process of atherosclerosis. ${ }^{4,5}$ Arterial stiffness is characterized by the loss of vessel elasticity and arterial compliance. The structural changes of the arterial wall are associated with aging, which are labeled by a decrease in the elastin content and collagen content of the arterial media. ${ }^{6}$

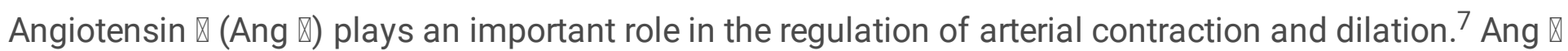
has been proved to induce arterial stiffness through structural and material remodeling.

Adherence of leukocytes to the vascular endothelium and their ensuing migration through the arterial wall into its intima where they instigate the migration of vascular smooth muscle cells (VSMCs) is one of the 
fundamental steps of atherosclerosis. ${ }^{8}$ Vascular cell adhesion molecule (VCAM) has been proved to induce leukocytes migration in the process of atherosclerosis. ${ }^{9}$

Our previous study found osteopontin (OPN) regulates migration and proliferation of VSMCs in animal models. ${ }^{10}$

Glutathione peroxidase (GPX) activity progressively decreased with decades of age in old people. Agerelated decline of GPX may represent a mechanism for the enhanced cardiovascular risk in the elderly population. ${ }^{11}$

Brachial-ankle pulse wave velocity (PWV) and ankle-brachial Index (ABI) are widely used in clinic for evaluating arterial stiffness. ${ }^{12,13}$ But there is no guide for choose of method for evaluating arterial stiffness in elderly population with different diseases, such as hypertension or diabetes mellitus (DM).

Our study aimed to compare the efficiency of PWV and $\mathrm{ABI}$ and investigate independent bio-markers for aging related arterial stiffness. We tested serum concentrations of Ang $\bigotimes, V C A M, O P N, G P X$ and evaluated indexes of arterial stiffness in elderly Chinese.

\section{Methods}

\subsection{Subjects and clinical parameters}

202 elderly individuals referred to Geriatrics department in Shanghai sixth people's hospital were evaluated. The inclusion criteria were: (1) aged above 65 years; (2) nonsmoker; (3) no alcoholic addiction. The exclusion criteria were: (1) tumor; (2) hepatic disease; (3) end-stage kidney disease (chronic kidney disease, CKD $\geq$ stage 4); (4) serious cardiovascular disease (acute myocardial infarction or New York Heart Association classification IV); (5) stroke within 3 months; (6) acute infection. A standard questionnaire was used to collect information, including age, sex, history of hypertension, history of DM, renal disease and use of antihypertensive or antidiabetic drugs or insulin. Hypertension was defined by a blood pressure of $140 / 90 \mathrm{~mm} \mathrm{Hg}$ or more, or a history of taking antihypertensive medications. DM was defined as fasting plasma glucose (FPG) equal or more than $7.0 \mathrm{mmol} / \mathrm{L}$, postprandial plasma glucose (PPG) equal or more than $11.1 \mathrm{mmol} / \mathrm{L}$, or the use of hypoglycemic agents. Body weight and body height were measured by professionals, and body mass index (BMI) was computed.

\subsection{Biological parameters}

Blood samples were obtained when the participants were in a fasting state in the morning. Biological variants were tested in the clinical laboratory of Shanghai Sixth People's Hospital. Serum levels of FPG, triglycerides (TG), total cholesterol (TC), low-density lipoprotein cholesterol (LDL), highdensity lipoprotein cholesterol (HDL) and creatinine were measured using an autoanalyzer (COBAS Integra 800, Roche 
Diagnostics, Basel, Switzerland). Hematocrit determination (HCT) was measured in blood samples using an automated blood analyzer (XE-5000, Sysmex, Kobe, Japan).

\subsection{Echocardiographic examination}

Echocardiography was performed at Medical Ultrasound department of Shanghai Jiaotong University affiliated Shanghai Sixth People's Hospital. Echocardiographic assessment was performed with an ACUSON Sequoia $^{\text {TM }} 512$ ultrasound system (Siemens Medical Solutions, Erlangen, Germany) equipped with an ultrabroad band frequency transducer $(3-8 \mathrm{MHz})$. The ejection fraction $(\mathrm{EF})$ was assessed using Simpson's biplane method.

\subsection{Brachial-ankle Pulse Wave Velocity (PWV)}

In this study, an Oscillometry-based device (BP-203RPE III; Colin

Omron, Co, Ltd, Tokyo, Japan) was used to measure the brachial-ankle PWV. After rested for at least ten minutes, each subject was laid in the supine position with PWV cuffs wrapped around both ankles and upper arms. Bilateral PWV were recorded for analysis after the examination of both sides. All measurements were performed by trained clinician with the manufacturer's guide.

\subsection{Ankle-brachial Index (ABI)}

$\mathrm{ABI}$ was defined as the ratio of ankle and brachial systolic blood pressure (SBP). Both ankle and brachial SBP were automatically and simultaneously measured by the validated device (VP-1000, Omron, Japan) on each side, the values of $A B I$ could be read directly from the device. Bilateral $A B I$ were used for further analyses. Measurements were performed by the same staff member, who was trained with the manufacturer's guide.

\subsection{Carotid Ultrasonography}

Carotid artery imaging was obtained by an experienced clinician using an ultrasound system (Acuson Sequoia 512 , Siemens, German). The probe frequency was $7.0 \mathrm{MHz}$ and the axis resolution was $0.1 \mathrm{~mm}$. Carotid intima media thickness (IMT), as the gap between the media-adventitia interface and lumenintima interface, was measured $1.0 \mathrm{~cm}$ distal to both common carotid artery (CCA) bifurcations. The measurement of IMT was carried out within a CCA region free of plaque.

\subsection{Assays for vascular endothelial cell adhesion molecule (VCAM),

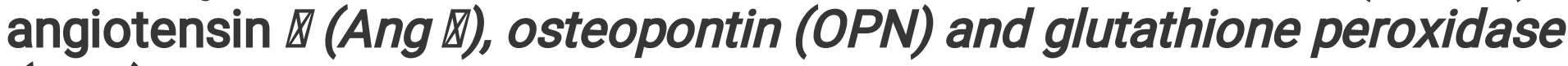 $(G P X)$}




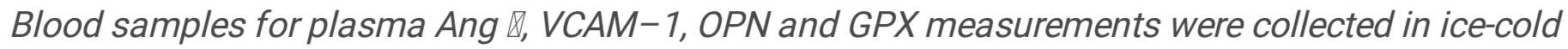
vacutainer tubes containing ethylenediaminetetraacetic acid to inhibit metalloproteases. A previous method was optimized for recovery and stability. The concentrations of Ang $\mathbb{Q}, V C A M-1$, OPN and GPX was measured by enzyme-linked immunosorbent assay (Rayto RT-6100, Rayto Life and Analytical Sciences Co., Shenzhen, China).

\section{Statistical analysis}

The unpaired Student's t-test, analysis of variance (ANOVA) and analysis of covariance were used for analysis of continuous variables. Quantitative data were presented as the mean \pm standard error (S. E. M.), while categorical data are presented as numbers. Associations between variables were conducted using Pearson correlation analysis, partial correlation analysis and multivariate linear stepwise regression analyses. Two sided $p$-values $<0.05$ were considered significant and $p$-values $<0.1$ were considered slightly significant. The software SPSS 19.0 for Windows (SPSS Inc., IBM, United States) was used for statistical analysis.

\section{Result}

\subsection{Clinical and laboratory characteristics}

Of the original 202 participants, 12 dropped out of the study and 21 were excluded because of various diseases, leaving 169 participants who were included in this cross-sectional study. Of the 169 participants, 119 were diagnosed with hypertension (70.4\%) and 62 were diagnosed with DM (36.7\%). There were 150 male participants (88.2\%), the mean age was $85.7 \pm 0.4$ years and the mean BMI was $23.8 \pm 0.3 \mathrm{~kg} / \mathrm{m}^{2}$. Laboratory parameters, $P W V, A B I$, and ultrasound parameters are reported in Table 1 .

\subsection{Comparison of clinical characteristics between hypertension group and normotension group}

To identify better method of evaluating arterial stiffness in elderly hypertensive patients, we compared $P W V$ and $A B I$ levels of hypertension group with those of normotension group. As shown in table 2, we observed higher TG concentrations and lower TC concentrations in hypertension group than in normotension group. Compared with normotensive participants, PWV levels of hypertensive patients were slightly higher in left limbs ( $2219 \pm 90 \mathrm{~cm} / \mathrm{s}$ v.s. $1970 \pm 66 \mathrm{~cm} / \mathrm{s}, P=0.090)$ and significantly higher in right limbs ( $2141 \pm 52 \mathrm{~cm} / \mathrm{s}$ v.s. $1932 \pm 67 \mathrm{~cm} / \mathrm{s}, P=0.023$ ) separately. The ABl levels of right limbs in hypertensive patients were lower than normotensive participants $(1.00 \pm 0.02$ v.s. $1.06 \pm 0.02, P=0.049)$, but no significant different $A B I$ levels were reported in left limbs. The levels of age, $B M I, F P G, L D L, H D L$, $H C T$, Creatinine, EF and IMT were not significantly different between hypertension group and normotension group. 


\subsection{Comparison of clinical characteristics between DM group and non- DM group}

To identify better method of evaluating arterial stiffness in elderly DM patients, we compared PWV and $A B I$ levels of DM group with those of non-DM group. FPG concentrations of DM patients were higher, while TC concentrations of DM patients were lower than those of non-DM participants. Compared with non- $D M$ participants, $A B$ l levels of DM patients were significantly lower in left limbs ( $0.96 \pm 0.02$ v.s. 1.05 $\pm 0.02, P=0.002)$ and slightly lower in right limbs $(0.99 \pm 0.03$ v.s. $1.03 \pm 0.02, P=0.071)$ separately. No difference was observed between PWV levels of DM patients and non-DM participants either in left limbs or in right limbs. TG concentrations were slightly higher and EF levels were slightly lower in DM patients compared to non-DM participants. The levels of age, BMI, FPG, LDL, HDL, HCT, creatinine and IMT were not significantly different between DM group and non-DM group. (Table 3)

\subsection{Peripheral expressions of candidate bio-markers for arterial stiffness}

For investigating potential bio-markers for arterial stiffness in elderly population, 86 candidates had the

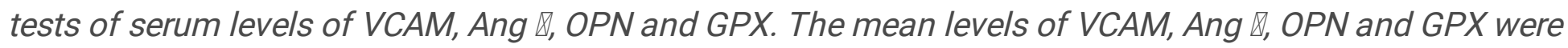
$318.8 \pm 7.1 \mathrm{ng} / \mathrm{ml}, 116.2 \pm 2.6 \mathrm{pg} / \mathrm{ml}, 33.8 \pm 0.7 \mathrm{ng} / \mathrm{ml}$ and $615.7 \pm 14.2 \mathrm{U} / \mathrm{L}$, separately. As shown in table $4 a$, the VCAM concentrations of hypertensive patients were significantly lower than normotensive

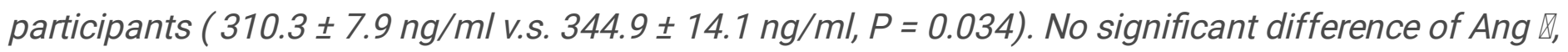

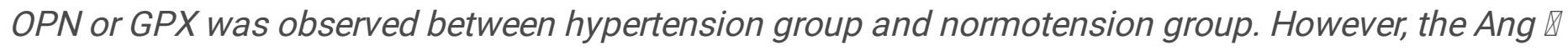
levels of DM patients were significantly lower than non-DM participants $(107.5 \pm 4.5 \mathrm{pg} / \mathrm{ml}$ v.s. $121.9 \pm$ $2.8 \mathrm{pg} / \mathrm{ml}, P=0.005)$. The levels of VCAM, OPN and GPX of DM patients were not significantly different from those of non-DM participants.

\subsection{Correlations between PWV and candidate bio-markers}

To investigate the roles of VCAM, Ang $甘, O P N$ and GPX in arterial stiffness in elderly population, we performed partial correlation analysis between PWV and candidate bio-markers. Both PWV of left limbs $(L-P W V)$ and $P W V$ of right limbs (R-PWV) were negatively correlated to VCAM $(R=-0.316, P=0.028 ; R=-$ $0.310, P=0.031$, separately) after adjusted for age, BMI, FPG, TC and HCT. OPN was negatively correlated

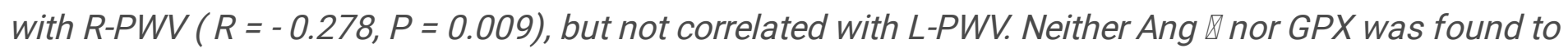
be correlated to PWV. (Table 5)

\subsection{Correlations between $\mathrm{ABI}$ and candidate bio-markers}

Partial correlation analysis between ABI and candidate bio-markers was performed. After adjusted for age, $B M I, F P G, T C$ and HCT, ABI of left limbs (L-ABI) was significantly correlated to Ang $\otimes(R=-0.381, P=$ 
0.010), but $A B$ I of right limbs (R-ABI) was only slightly correlated to Ang $\otimes(R=-0.234, P=0.082)$. OPN was negatively correlated with $R-A B I(R=-0.303, P=0.005)$, but not correlated with L-ABI. Neither VCAM nor GPX was found to be correlated to $A B I$. ( Table 6)

\subsection{Bio-markers for predicting arterial stiffness in regression models}

Since VCAM and OPN were found to be correlated to PWV, linear regression analysis of PWV with variate of age, BMI, FPG, TC, HCT, VCAM and OPN were performed. Only VCAM was included in the regression models of L-PWV and R-PWV (Beta $=-0.307, P=0.048 ;$ Beta $=-0.358, P=0.020$, separately). Table 7

Linear regression analysis of $A B I$ with variate of age, BMI, FPG, TC, HCT, Ang $\unrhd$ and OPN were performed. $O P N$ was included both in the regression models of $L-A B I$ and $R-A B I$ (Beta = - 0.320, $P=0.039 ; B e t a=-$ $0.290, P=0.011$, separately). In the second model of $R-A B I$, both OPN and FPG were included. However, Ang $\otimes$ was not included in any model of $A B$. Table 8

\section{Discussion}

Population aging leads to more patients suffered from diseases related to arterial stiffness, such as cardiovascular disease, cerebral vascular disease and diabetes nephropathy. Not only high plasm glucose, lipid and high blood pressure, but also aging itself can cause arterial stiffness. ${ }^{14}$ Our study included subjects at an average age of 85 years old, which represented elderly Chinese population.

We found PWV levels were elevated in both limbs of hypertensive patients compared with nonhypertensive subjects. However, only $A B I$ of right limb was slightly lower in hypertensive patients. We deduce PWV might be better method for evaluating arterial stiffness in hypertensive patients.

$A B$ l levels of DM patients were lower in both limbs compared with non-DM participants. But no significant difference of PWV was observed between DM patients and non-DM participants. ABI might be better method for evaluating DM related vascular disease.

The mechanisms of arterial stiffness are not single. There are plenty of studies revealing the potential

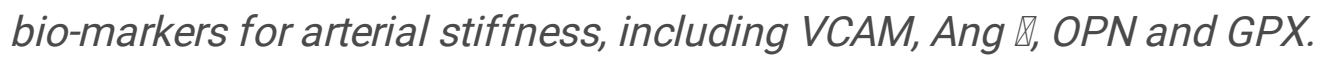

Recently, the serum VCAM level was reported to be elevated in patients with peripheral artery disease and systemic sclerosis. ${ }^{15,16}$ However, we found the serum VCAM levels of hypertensive patients were lower than normotensive participants. In previous published study, serum VCAM levels were not found to be elevated in pulmonary hypertension or gestational hypertension, and low VCAM level might lead to fetal growth restriction. ${ }^{16,17}$ Our study found VCAM was negatively correlated with PWV in the total sample of elderly subjects. VCAM was the only factor included in the model for predicting PWV in our further linear regression analysis of PWV with variate of age, BMI, FPG, TC, HCT, VCAM and OPN. We deduced VCAM might be an independent biomarker for arterial stiffness caused by hypertension. 
Renin-Angiotensin-Aldosterone System has been proved to play important roles in arterial remodeling of

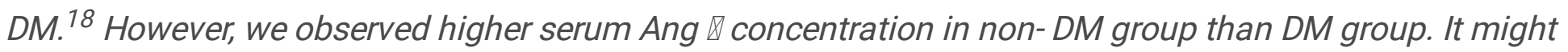
be associated with the advocated use of angiotensin converting enzyme inhibitors and Ang $₫$ receptor blockers as blood pressure lowering agents in DM patients. ${ }^{19}$ We also found Ang $\otimes$ was related to $L-A B I$

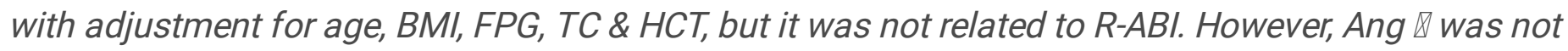
included in any model of biomarkers for $A B I$ in further linear regression analysis.

Interestingly, we found OPN was negatively correlated to PWV and $A B I$ in right limbs, but not left limbs. Moreover, OPN was included in all of the regression models of biomarkers for $A B I$, both in left limbs and right limbs. Although OPN was not found to be differently expressed in serum of hypertension or DM patients in our study, it might be an independent bio-marker for predicting arterial stiffness in elderly population. This finding is consistent with a former published animal study, which reported that OPN exerts stage-specific roles in arteriosclerosis by regulating vascular calcification and fibrosis. 20

\section{Conclusion}

Differential use of PWV and ABI should be considered in elderly with hypertension and/or DM. Serum levels of VCAM and OPN might be independent bio-markers for predicting arterial stiffness.

\section{Abbreviations}

PWV: brachial-ankle pulse wave velocity; ABI: ankle-brachial Index; VCAM: vascular cell adhesion

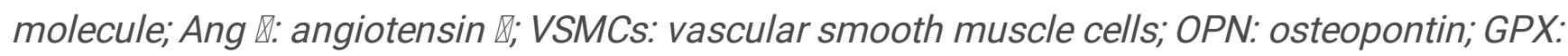
glutathione peroxidase; DM: diabetes mellitus; FPG: fasting plasma glucose; PPG: postprandial plasma glucose; BMI: body mass index; TG: triglycerides; TC: total cholesterol; LDL: low-density lipoprotein cholesterol; HDL: highdensity lipoprotein cholesterol; HCT: hematocrit determination; EF: ejection fraction; SBP: systolic blood pressure; IMT: carotid intima media thickness; CCA: common carotid artery; L-PWV: $P W V$ of left limbs; R-PWV: PWV of right limbs; $L-A B I$ : $A B I$ of left limbs; $R-A B I$ : $A B I$ of right limbs.

\section{Declarations}

\section{Ethics approval and consent to participate}

The protocol of the study was approved by the ethics committee of Shanghai Jiaotong University Affiliated Sixth People's Hospital (Shanghai, China). Each participate gave written informed consent to the procedure.

\section{Consent for publication}

Not applicable. 


\section{Availability of data and materials}

The datasets used and/or analysed during the current study are de-identified but not available because of privacy protection.

\section{Competing interests}

The authors declare no conflict of interest in relation to the current study.

\section{Funding}

This study was supported by Shanghai Shen Kang Hospital Development Center Clinical Research Training Project (Grant No. SHDC12017X21). The funding body had no role in the study design; collection, analysis, and interpretation of data; writing of the report; or decision to submit the article for publication.

\section{Authors' contributions}

Guoxiang Fu and Yuan Zhong contributed equally to the work.

Guoxiang Fu provided funding and administrative support. Yuan Zhong recruited the participants. Chanchan Xu took responsibility for all aspects of the reliability and freedom from bias of the data presented. Chanchan Xu was a major contributor in writing the manuscript and took responsibility for research design. Chanchan Xu, Guoxiang Fu and Fengfeng Pan were involved in data collection. Chanchan Xu and Guoxiang Fu were involved in statistical analysis. All authors read and approved the final manuscript.

\section{Acknowledgements}

We gratefully acknowledge the invaluable assistance offered by the physicians in the Geriatrics Department of Shanghai Jiaotong University affiliated Shanghai Sixth people's Hospital.

\section{References}

1. Naghavi M, Abajobir AA, Abbafati C, Abbas KM, Abd-Allah F, Abera SF. Global, regional, and national age-sex specifc mortality for 264 causes of death, 1980-2016: a systematic analysis for the Global Burden of Disease Study 2016. Lancet. 2017;390(10100):1151-210.

2. N. Saji, K. Toba, T. Sakurai, Cerebral small vessel disease and arterial stiffness: tsunami effect in the brain. Pulse. 2016;3 (3-4):182-189.

3. Antonino Tuttolomondo, Rosaria Pecoraro, Domenico Di Raimondo, Riccardo Di Sciacca, Baldassare Canino, Valentina Arnao, et al. Immune-inflammatory markers and arterial stiffness indexes in 
subjects with acute ischemic stroke with and without metabolic syndrome. Diabetology \& Metabolic Syndrome. 2014;6:28.

4. Boutouyrie P, Tropeano Al, Asmar R, Gautier I, Benetos A, Lacolley P. Aortic stiffness is an independent predictor of primary coronary events in hypertensive patients: a longitudinal study. Hypertension. 2002;39:10-15.

5. Arnett DK, Boland LL, Evans GW, Riley W, Barnes R, Tyroler HA. Hypertension and arterial stiffness: the Atherosclerosis Risk in Communities Study. ARIC Investigators. Am J Hypertens. 2000;13:317323.

6. Nilsson PM, Boutouyrie P, Cunha P, Kotsis V, Narkiewicz K, Parati G, et al. Early vascular ageing in translation: from laboratory investigations to clinical applications in cardiovascular prevention. $J$ Hypertens. 2013; 8:1517-26.

7. Barrett-O'Keefe Z, Witman MA, McDaniel J, Fjeldstad AS, Trinity JD, Ives SJ. Angiotensin II potentiates a-adrenergic vasoconstriction in the elderly. Clin Sci (Lond). 2013;124:413-22.

8. Hunt BJ, Jurd KM. Endothelial cell activation. BMJ. 1998;316:1328-9.

9. Deanfield JE, Halcox JP, Rabelink TJ. Endothelial function and dysfunction: testing and clinical relevance. Circulation. 2007;115:1285-95.

10. Guoxiang Fu, Chanchan Xu, Yuan Zhong, Dingliang Zhu, Pingjin Gao. Aldosterone-induced steopontin expression in vascular smooth muscle cells involves MR, ERK, and p38 MAPK. Endocrine. 2012;42(3):676-83.

11. Daniele Pastori, Pasquale Pignatelli, Alessio Farcomeni, Danilo Menichelli, Cristina Nocella, Roberto Carnevale, et al. Aging-Related Decline of Glutathione Peroxidase 3 and Risk of Cardiovascular Events in Patients With Atrial Fibrillation. J Am Heart Assoc. 2016;5(9).

12. Ohkuma T, Tomiyama H, Ninomiya T. Proposed Cutoff Value of Brachial-Ankle Pulse Wave Velocity for the Management of Hypertension. Circ J. 2017;81(10):1540-1542.

13. Janelle M, Guirguis-Blake, Corinne V Evans, Nadia Redmond, Jennifer S Lin. Screening for Peripheral Artery Disease Using the Ankle-Brachial Index Updated Evidence Report and Systematic Review for the US Preventive Services Task Force. JAMA. 2018;320(2):184-196.

14. O'Rourke MF, Safar ME, Dzau V. The Cardiovascular Continuum extended: aging effects on the aorta and microvasculature. Vasc Med. 2010;15:461-8.

15. Edlinger C, Lichtenauer M, Wernly B, Pistulli R, Paar V, Prodinger C, et al. Disease-specific characteristics of vascular cell adhesion molecule-1 levels in patients with peripheral artery disease. Heart Vessels. 2019;34(6):976-983.

16. Thakkar V, Patterson KA, Stevens W, Wilson M, Roddy J, Sahhar J, et al. Increased serum levels of adhesion molecules ICAM-1 and VCAM-1 in systemic sclerosis are not specific for pulmonary manifestations. Clin Rheumatol. 2018,37(6):1563-1571.

17. Madazli R, Benian A, Ilvan S, Calay Z. Placental apoptosis and adhesion molecules expression in the placenta and the maternal placental bed of pregnancies complicated by fetal growth restriction with and without pre-eclampsia. J Obstet Gynaecol. 2006;26(1):5-10. 
18. Joseph JJ, Echouffo Tcheugui JB, Effoe VS, Hsueh WA, Allison MA, Golden SH. Renin-AngiotensinAldosterone System, Glucose Metabolism and Incident Type 2 Diabetes Mellitus: MESA. J Am Heart Assoc. 2018;7(17):e009890.

19. Ponirakis G, Petropoulos IN, Alam U, Ferdousi M, Asghar O, Marshall A, et al. Hypertension Contributes to Neuropathy in Patients With Type 1 Diabetes. Am J Hypertens. 2019;32(8):796-803.

20. Shao JS, Sierra OL, Cohen R, Mecham RP, Kovacs A, Wang J, et al. Vascular calcification and aortic fibrosis: a bifunctional role for osteopontin in diabetic arteriosclerosis. Arterioscler Thromb Vasc Biol. 2011;31(8):1821-33.

\section{Tables}




\section{Table 1. Clinical characteristics of total subjects}

\begin{tabular}{ccc}
\hline Clinical characteristics & Mean value & SE \\
\hline FPG $(\mathrm{mmol} / \mathrm{L})$ & 5.64 & 0.13 \\
$\mathrm{TG}(\mathrm{mmol} / \mathrm{L})$ & 1.89 & 0.12 \\
$\mathrm{TC}(\mathrm{mmol} / \mathrm{L})$ & 3.36 & 0.12 \\
$\mathrm{LDL}(\mathrm{mmol} / \mathrm{L})$ & 2.16 & 0.06 \\
$\mathrm{HDL}(\mathrm{mmol} / \mathrm{L})$ & 1.09 & 0.03 \\
$\mathrm{HCT}(\%)$ & 38.4 & 0.5 \\
Creatinine $(\mathrm{umol} / \mathrm{L})$ & 89.1 & 3.0 \\
L- PWV (cm/s) & 2145 & 67 \\
$\mathrm{R}-\mathrm{PWV} \mathrm{(cm/s)}$ & 2079 & 42 \\
$\mathrm{~L}-\mathrm{ABI}$ & 1.01 & 0.01 \\
$\mathrm{R}-\mathrm{ABI}$ & 1.02 & 0.01 \\
$\mathrm{EF}(\%)$ & 63 & 0.69 \\
IMT $(\mathrm{mm})$ & 0.86 & 0.01 \\
\hline
\end{tabular}

FPG: fasting plasma glucose; TG: triglycerides, TC: total cholesterol; LDL: low-density lipoprotein cholesterol; HDL: high-density lipoprotein cholesterol; HCT: red blood cell specific volume ; PWV: pulse wave velocity; L- PWV: PWV in left limbs; R- PWV: PWV in right limbs; ABI: ankle brachial index; $L-A B I: A B I$ in left limbs; $R-A B I: A B I$ in right limbs; $E F$ : ejection fraction; IMT: intima media thickness 
Table 2. Clinical characteristics between hypertensive patients and normotensive participants

\begin{tabular}{cccc}
\hline Clinical parameters & Hypertension & Normotension & P-value \\
\hline $\mathrm{n}$ & 119 & 50 & $/$ \\
Age (years) & $86.1 \pm 0.4$ & $84.8 \pm 0.9$ & 0.156 \\
BMI & $23.7 \pm 0.3$ & $24.0 \pm 0.5$ & 0.670 \\
FPG (mmol/L) & $5.76 \pm 0.18$ & $5.40 \pm 0.05$ & 0.215 \\
TG (mmol/L) & $2.09 \pm 0.16^{*}$ & $1.48 \pm 0.17^{*}$ & 0.018 \\
TC (mmol/L) & $3.09 \pm 0.16^{*}$ & $3.92 \pm 0.18^{*}$ & 0.002 \\
LDL (mmol/L) & $2.11 \pm 0.08$ & $2.30 \pm 0.11$ & 0.194 \\
HDL (mmol/L) & $1.07 \pm 0.05$ & $1.10 \pm 0.06$ & 0.760 \\
HCT (\%) & $38.0 \pm 0.6$ & $39.3 \pm 0.7$ & 0.607 \\
Creatinine ( $\mu$ mol/L) & $92.3 \pm 3.8$ & $83.1 \pm 4.5$ & 0.141 \\
L -PWV (cm/s) & $2219 \pm 90^{*}$ & $1970 \pm 66{ }^{*}$ & 0.090 \\
R -PWV (cm/s) & $2141 \pm 52^{*}$ & $1932 \pm 67 *$ & 0.023 \\
L -ABI & $1.00 \pm 0.02$ & $1.03 \pm 0.02$ & 0.313 \\
R -ABI & $1.00 \pm 0.02 *$ & $1.06 \pm 0.02 *$ & 0.049 \\
EF (\%) & $63.4 \pm 0.6$ & $62.3 \pm 1.9$ & 0.534 \\
IMT (mm) & $0.85 \pm 0.01$ & $0.86 \pm 0.03$ & 0.734 \\
\hline
\end{tabular}

$=P<0.05, " P<0.1$

BMI: body mass index; FPG: fasting plasma glucose; TG: triglycerides, TC: total cholesterol; LDL: low-density lipoprotein cholesterol; HDL: high-density lipoprotein cholesterol; HCT: red blood cell specific volume; PWV: pulse wave velocity; L- PWV: PWV in left limbs; R- PWV: PWV in right limbs; ABI: ankle brachial index; $L-A B I: A B I$ in left limbs; $R-A B I$ : $A B I$ in right limbs; $E F$; ejection fraction; IMT: intima media thickness 
Table 3. Clinical characteristics between DM patients and non- DM participants

\begin{tabular}{cccc}
\hline Clinical parameters & Diabetes Mellitus & Non- Diabetes Mellitus & P-value \\
\hline$n$ & 62 & 107 & $/$ \\
Age (years) & $85.9 \pm 0.6$ & $85.5 \pm 0.5$ & 0.638 \\
BMI & $23.7 \pm 0.5$ & $23.8 \pm 0.3$ & 0.818 \\
FPG (mmol/L) & $6.69 \pm 0.34^{*}$ & $5.18 \pm 0.05^{*}$ & 0.000 \\
TG (mmol/L) & $2.21 \pm 0.23^{*}$ & $1.73 \pm 0.13^{*}$ & 0.062 \\
TC (mmol/L) & $2.90 \pm 0.22^{*}$ & $3.59 \pm 0.15^{*}$ & 0.008 \\
LDL (mmol/L) & $2.13 \pm 0.12$ & $2.20 \pm 0.08$ & 0.624 \\
HDL (mmol/L) & $1.04 \pm 0.07$ & $1.11 \pm 0.04$ & 0.377 \\
HCT & $37.0 \pm 0.8$ & $39.1 \pm 0.5$ & 0.607 \\
Creatinine ( $\mu$ mol/L) & $94.6 \pm 5.6$ & $86.2 \pm 3.4$ & 0.180 \\
L- PWV & $2141 \pm 90$ & $2148 \pm 92$ & 0.964 \\
R- PWV & $2126 \pm 71$ & $2053 \pm 52$ & 0.405 \\
L- ABI & $0.96 \pm 0.02 *$ & $1.05 \pm 0.02 *$ & 0.002 \\
R- ABI & $0.99 \pm 0.03 *$ & $1.03 \pm 0.02 *$ & 0.076 \\
EF $(\%)$ & $61.7 \pm 1.4 *$ & $64.2 \pm 0.6 *$ & 0.071 \\
IMT (mm) & $0.87 \pm 0.02$ & $0.85 \pm 0.01$ & 0.359 \\
\hline
\end{tabular}

* $P<0.05,{ }^{*} P<0.1$

DM: Diabetes Mellitus; BMI: body mass index; FPG: fasting plasma glucose; TG:

triglycerides, TC: total cholesterol; LDL: low-density lipoprotein cholesterol; HDL: high-density lipoprotein cholesterol; HCT: red blood cell specific volume; PWV: pulse wave velocity; L- PWV: PWV in left limbs; R- PWV: PWV in right limbs; $A B I$ : ankle brachial index, $L-A B I: A B I$ in left limbs; R-ABI: $A B I$ in right limbs; $E F$ : ejection fraction; IMT: intima media thickness 
Table 4a. Serum levels of VCAM, Ang II, OPN and GPX in hypertension group and normotension group

\begin{tabular}{cccc}
\hline Laboratory Variable & Hypertension & Normotension & P-value \\
\hline VCAM $(\mathrm{ng} / \mathrm{ml})$ & $310.3 \pm 7.9^{*}$ & $344.9 \pm 14.1^{*}$ & 0.034 \\
Ang II $(\mathrm{pg} / \mathrm{ml})$ & $115.4 \pm 3.2$ & $118.8 \pm 3.9$ & 0.575 \\
OPN $(\mathrm{ng} / \mathrm{ml})$ & $33.29 \pm 0.73$ & $35.33 \pm 1.85$ & 0.220 \\
GPX $(\mathrm{U} / \mathrm{L})$ & $620.5 \pm 16.1$ & $600.7 \pm 30.3$ & 0.552 \\
\hline
\end{tabular}

${ }^{*} \mathrm{P}<0.05$.

VCAM: vascular endothelial cell adhesion molecule; Ang II : angiotensin II; OPN:

osteopontin; GPX: glutathione peroxidase

Table 4b. Serum levels of VCAM, Ang II, OPN and GPX in DM group and non- DM group

\begin{tabular}{cccc}
\hline Laboratory Variable & Diabetes Mellitus & Non- Diabetes Mellitus & P-value \\
\hline VCAM $(\mathrm{ng} / \mathrm{ml})$ & $311.3 \pm 12.3$ & $323.6 \pm 8.5$ & 0.396 \\
Ang II $(\mathrm{pg} / \mathrm{ml})$ & $107.5 \pm 4.5^{*}$ & $121.9 \pm 2.8^{*}$ & 0.005 \\
OPN $(\mathrm{ng} / \mathrm{ml})$ & $33.92 \pm 0.92$ & $33.70 \pm 1.03$ & 0.881 \\
GPX $(\mathrm{U} / \mathrm{L})$ & $627.7 \pm 23.6$ & $607.8 \pm 17.8$ & 0.495 \\
\hline
\end{tabular}

${ }^{*} \mathrm{P}<0.05$.

DM: Diabetes Mellitus; VCAM: vascular endothelial cell adhesion molecule; Ang II:

angiotensin II ; OPN: osteopontin; GPX: glutathione peroxidase 
Table 5. Pearson correlation analysis between PWV and candidate bio- markers with adjustment for age, BMI, FPG, TC and HCT.

\begin{tabular}{ccccc}
\hline \multirow{2}{*}{ Variable } & \multicolumn{2}{c}{ L- PWV } & \multicolumn{2}{c}{ R- PWV } \\
\cline { 2 - 5 } & $\mathrm{R}$ & $\mathrm{P}$-value & $\mathrm{R}$ & $\mathrm{P}$-value \\
\hline VCAM & $-0.316^{*}$ & 0.028 & $-0.310^{*}$ & 0.031 \\
Ang II & -0.141 & 0.203 & -0.004 & 0.489 \\
OPN & -0.084 & 0.240 & $-0.278^{*}$ & 0.009 \\
GPX & -0.083 & 0.314 & -0.094 & 0.290 \\
\hline
\end{tabular}

${ }^{*} \mathrm{P}<0.05$.

$\mathrm{R}$ : correlation coefficient

PWV: pulse wave velocity; L- PWV: PWV in left limbs; R- PWV: PWV in right limbs; VCAM: vascular endothelial cell adhesion molecule; Ang II: angiotensin II; OPN: osteopontin; GPX: glutathione peroxidase; FPG: fasting plasma glucose; TC: cholesterol; BMI: body mass index; HCT: red blood cell specific volume

Table 6. Pearson correlation analysis between laboratory variable and $\mathrm{ABI}$ with adjustment for age, BMI, FPG, TC \& HCT .

\begin{tabular}{ccccc}
\hline \multirow{2}{*}{ Variable } & \multicolumn{2}{c}{$\mathrm{L}-\mathrm{ABI}$} & \multicolumn{2}{c}{$\mathrm{R}$-ABI } \\
\cline { 2 - 5 } & $\mathrm{R}$ & $\mathrm{P}$-value & $\mathrm{R}$ & $\mathrm{P}$-value \\
\hline VCAM & -0.171 & 0.155 & 0.063 & 0.356 \\
Ang II & $-0.381^{*}$ & 0.010 & $-0.234^{*}$ & 0.082 \\
OPN & -0.145 & 0.112 & $-0.303^{*}$ & 0.005 \\
GSH & 0.148 & 0.191 & -0.007 & 0.483 \\
\hline
\end{tabular}

${ }^{*} \mathrm{P}<0.05,{ }^{*} \mathrm{P}<0.1$.

$\mathrm{R}$ : correlation coefficient;

ABI: ankle brachial index; VCAM: vascular endothelial cell adhesion molecule; Ang II: angiotensin II; OPN: osteopontin; GPX: glutathione peroxidase; FPG: fasting plasma glucose; TC: cholesterol; BMl: body mass index; HCT: red blood cell specific volume 
Table 7.

Linear regression analysis of L- PWV with variate of age, BMI, FPG, TC, HCT, VCAM and OPN

\begin{tabular}{cccc}
\hline Variate & Beta & P-value & State \\
\hline VCAM & $-0.307^{\star}$ & 0.048 & included \\
age & -0.109 & 0.486 & excluded \\
BMI & -0.050 & 0.751 & excluded \\
FPG & 0.129 & 0.400 & excluded \\
TC & 0.034 & 0.834 & excluded \\
HCT & -0.097 & 0.529 & excluded \\
OPN & 0.009 & 0.952 & excluded \\
\hline
\end{tabular}

Linear regression analysis of R- PWV with variate of age, BMI, FPG, TC, HCT, VCAM and OPN

\begin{tabular}{cccc}
\hline Variate & Beta & P-value & State \\
\hline VCAM & $-0.358^{*}$ & 0.020 & included \\
age & 0.108 & 0.481 & excluded \\
BMI & -0.043 & 0.779 & excluded \\
FPG & 0.154 & 0.303 & excluded \\
TC & -0.029 & 0.855 & excluded \\
HCT & 0.036 & 0.813 & excluded \\
OPN & -0.227 & 0.13 & excluded \\
\hline
\end{tabular}

" $P<0.05$. Beta: standardized coefficients for multivariable regression model.

PWV: pulse wave velocity; L- PWV: PWV in left limbs; R- PWV: PWV in right limbs; FPG: fasting plasma glucose; TC: cholesterol; BMI: body mass index; HCT: red blood cell specific volume; VCAM: vascular endothelial cell adhesion molecule; OPN: osteopontin 
Table 8.

Linear regression analysis of $\mathrm{L}-\mathrm{ABI}$ with variate of age,

BMI, FPG, TC, HCT, Ang II and OPN

\begin{tabular}{cccc}
\hline Variate & Beta & P-value & State \\
\hline OPN & $-0.320^{*}$ & 0.039 & included \\
age & 0.043 & 0.779 & excluded \\
BMI & 0.041 & 0.787 & excluded \\
FPG & -0.201 & 0.183 & excluded \\
TC & 0.091 & 0.549 & excluded \\
HCT & -0.007 & 0.963 & excluded \\
Ang II & -0.198 & 0.203 & excluded \\
\hline
\end{tabular}

Linear regression analysis of $\mathrm{R}-\mathrm{ABI}$ with variate of age, BMI, FPG, TC, HCT, VCAM and OPN

\begin{tabular}{|c|c|c|c|c|c|c|}
\hline \multirow[t]{2}{*}{ Variate } & \multicolumn{3}{|c|}{ Model 1} & \multicolumn{3}{|c|}{ Model 2} \\
\hline & Beta & P-value & State & Beta & $\mathrm{P}$-value & State \\
\hline OPN & $-0.290^{*}$ & 0.011 & included & $-0.274^{*}$ & 0.013 & included \\
\hline age & -0.195 & 0.082 & excluded & -0.169 & 0.124 & excluded \\
\hline BMI & -0.039 & 0.831 & excluded & -0.034 & 0.745 & excluded \\
\hline FPG & -0.257 & 0.020 & excluded & $-0.257^{*}$ & 0.020 & included \\
\hline $\mathrm{TC}$ & -0.036 & 0.756 & excluded & -0.004 & 0.972 & excluded \\
\hline HCT & -0.122 & 0.278 & excluded & -0.127 & 0.243 & excluded \\
\hline Ang II & 0.000 & 0.998 & excluded & -0.008 & 0.941 & excluded \\
\hline
\end{tabular}

${ }^{*} \mathrm{P}<0.05$. Beta: standardized coefficients for multivariable regression model. $A B I$ : ankle brachial index; $L-A B I: A B I$ in left limbs; $R-A B I: A B I$ in right limbs; FPG: fasting plasma glucose; TC: cholesterol; BMI: body mass index; HCT: red blood cell specific volume; Ang II : angiotensin II; OPN: osteopontin; 